\title{
Fourier Transform Infrared Imaging Analysis of Cancellous Bone in Alendronate- and Raloxifene-Treated Osteopenic Sheep
}

\author{
Ericka F. Calton ${ }^{\mathrm{a}, \mathrm{b}}$ Jennifer MacLeay ${ }^{\mathrm{c}}$ Adele L. Boskey $^{\mathrm{b}}$ \\ ${ }^{a}$ Grove School of Engineering, City College and the Graduate Center of the City University of New York, and \\ ${ }^{b}$ Mineralized Tissue Laboratory, Hospital for Special Surgery, New York, N.Y., and 'Hill's Pet Nutrition, \\ Topeka, Kans., USA
}

\section{Key Words}

Osteoporosis · Antiresorptive treatment • Fourier transform infrared spectroscopy · Sheep

\begin{abstract}
Fourier transform infrared imaging spectroscopy (FTIRI)assessed bone composition parameters (mineral content, collagen maturity, crystal size and perfection, and carbonate content) describe bone quality and correlate to bone fracture risk. The challenge with studying bone quality in patients treated with antiresorptive drugs such as bisphosphonates (e.g., alendronate) and selective estrogen receptor modulators (SERMs) (e.g. raloxifene) is being able to test bone mechanical performance and material properties preand posttreatment. The purpose of this study was to evaluate the FTIRI changes in a large animal model of osteoporosis (female sheep with dietary induced metabolic acidosis; MA). Previous studies have investigated the relationship between bone material properties and bone strength in humans and smaller animals and have shown that changes in compositional properties influence fracture risk. Here we characterize the MA model at 6 and 12 months, demonstrate the loss of bone and changes in compositional properties, and show that 6 months of treatment with both antiresorptives ameliorate the bone loss as assessed by bone mineral density and FTIRI. This preliminary data suggest that the MA sheep
\end{abstract}

model allows investigation of whether drug treatments preserve bone properties that exist at the time of treatment or if they induce further beneficial changes.

Copyright $\odot 2011$ S. Karger AG, Basel

\section{Introduction}

Osteoporosis is a progressive disease of bone loss [Cummings et al., 2002a] resulting in an increased risk of fracture that is not necessarily a normal consequence of aging [Boskey and Coleman, 2010]. Osteoporosis, which affects trabecular bone most noticeably [Goldstein, 1987], primarily affects Caucasian and Asian women [Harris et al., 1995] but can occur in men and women of all ethnic-

\section{Abbreviations used in this paper}

ALN alendronate

BMD bone mineral density

BP bisphosphonate

DXA dual energy X-ray absorptiometry

FTIRI fourier transform infrared imaging spectroscopy

MA metabolic acidosis

RLX raloxifene

SERM selective estrogen receptor modulator

\section{KARGER}

๑) 2011 S. Karger AG, Basel

Fax +4161306 1234

E-Mail karger@karger.ch

www.karger.com
Accessible online at: www.karger.com/cto
Dr. Adele Boskey

Mineralized Tissue Laboratory, Hospital for Special Surgery

535 E 70 th Street

New York, NY 10021 (USA)

Tel. +1 212606 1453, E-Mail Boskeya@ @ss.edu 
ities. Osteoporosis causes changes in bone composition which contribute to fracture risk [Gourion-Arsiquaud et al., 2009]. Osteoporotic changes in bone include decreased bone mineral density (BMD) and trabecular thinning and loss of trabecular connectivity as well as changes in collagen composition [Boskey and Coleman, 2010].

Throughout life, bone is continuously remodeled (resorbed and reformed); where these processes are unbalanced, bone loss and osteoporosis may occur. The trabeculae in osteoporotic bone have decreased mineral content, increased crystallinity, and increased collagen maturity [Mendelsohn et al., 2005] compared to nonosteoporotic healthy (normal) bone. Treatments for osteoporosis should be evaluated based on how effectively they restore bone material properties to 'normal' levels. When patients are diagnosed with osteoporosis by DXA scores indicating decreased BMD relative to normal sexmatched 25-year-olds or by the incidence of fractures, physicians have a choice of therapeutic agents to restore BMD. BMD correlates weakly with fracture risk, and a large reduction in fracture risk may be associated with little or no detectable change in BMD [Cummings et al., $2002 \mathrm{a}, \mathrm{b}]$. To the best of our knowledge, there is no clinical method that has a sufficiently high resolution to observe changes in both cancellous and cortical bone before, during, and after osteoporosis drug intervention. Therefore, a preclinical animal model is needed to allow evaluation of the effects of therapy on both compositional and mechanical properties. The FDA requires testing of therapies in a rodent model and in a second animal model. Animal models of osteoporosis are reviewed elsewhere [Newman et al., 1995; Turner et al., 2001], and each has limitations. Rodents do not have Haversian remodeling and do not lose bone with age or with estrogen deficiency; canines and nonhuman primates which do show age- and estrogen-related bone loss are expensive and difficult to work with. Sheep, on the other hand, are docile, have an estrous cycle, and are relatively less costly, and they exhibit age-related, glucocorticoid-related, and estrogen-related bone loss [Turner, 2002].

Both estrogen reduction in menopausal women and metabolic acidosis (MA) in humans and sheep induce bone loss and decreases in mechanical strength suggesting MA can provide a model of osteoporosis in ovaryintact sheep in a relatively short time span. In humans, MA results in a decrease in total body calcium since calcium excretion exceeds calcium absorption from the gut [Wiederkehr and Krapf, 2001]. With dietary induced metabolic acidosis, there is a high negative cation-anion difference; bone formation is decreased and bone formation is increased in sheep as the metabolism responds to maximize the availability of hydroxyl ions as a buffer [Wiederkehr and Krapf, 2001; MacLeay et al., 2004a, b]. Sheep also respond to drugs used in humans; for example, bone loss observed in mandibles of ovariectomized ewes has been shown to respond to raloxifene (RLX), a selective estrogen receptor modulator (SERM) [Turner et al., 2002], with increased bone formation. This is consistent with findings that estrogen can have a direct effect on bone formation [Bonnelye and Aubin, 2005; Boskey, unpubl. data]. Material, and especially compositional, properties of sheep bones in models of osteoporosis have not been extensively described.

Fourier transform infrared imaging spectroscopy (FTIRI) is a vibrational spectroscopy technique that is sensitive to the chemical composition of bone and can be used to quantitate bone material properties and their distribution. Mean values and distributions of FTIRI-derived parameters have been established and used to compare human osteoporotic bones and bone biopsies from osteoporotic patients treated with alendronate (ALN) [Boskey et al., 2005], RXL [Faibish et al., 2006], and risedronate [Durchschlag et al., 2006]. Previous studies indicate decreased heterogeneity with ALN treatment [Boskey et al., 2005] relative to nontreated controls; however, pre- and posttreatment biopsies are difficult to obtain, especially if the therapy prevents further fracture, preventing mechanical testing and compositional analysis of samples from the same patient over time.

The purpose of this study was to evaluate the bone composition of osteopenic sheep after the progression of bone loss by MA ( 6 months) and after 6 months of drug treatment (12 months) for bone loss. This study addresses the hypothesis that a SERM (RLX) better restores bone quality as defined using FTIRI than a bisphosphonate (BP) (ALN) in sheep with normal estrogen levels. Bone quality was assessed by FTIRI spatially resolved analysis of trabecula integrity.

\section{Materials and Methods}

Skeletally mature (4-7 years old; $169 \pm 29$ lb) RambouilletColumbia cross-ewes $(n=18)$ were administered a diet to induce metabolic acidosis [MacLeay et al., 2004a, b]. At 6 months, with bone loss similar to that seen in human osteopenia as measured by dual energy X-ray absorptiometry (DXA), each sheep was anesthetized and a permanent cannula was inserted into the abomasums, threaded under the subcutaneous skin, and secured to the outside of the ewe for ease of access. Daily treatment with 
RLX, ALN, or saline (vehicle) ( $\mathrm{n}=6$ sheep/treatment group) was administered for 6 months via the permanent cannulae until sacrifice at 12 months (IACUC-approved protocol, Colorado State University) for sample harvest. Sheep were anesthetized for DXA scans of the lumbar spine at 3 time points $(0,6$, and 12 months) using a Hologic Delphi QDR DXA (Hologic). Six untreated agematched sheep used for other non-bone-related studies served as normal controls.

Transiliac crest biopsies (8-mm diameter) were obtained after 6 months of MA and at 12 months from bilateral sites under an IACUC-approved protocol. Iliac crest biopsies were bisected axially. Half of the biopsy was dehydrated in increasing concentrations of ethanol $(70-100 \% \mathrm{v} / \mathrm{v})$ and embedded in polymethylmethacrylate. Three 2 - $\mu \mathrm{m}$-thick sections per specimen block were placed on $\mathrm{BaF}_{2}$ infrared windows for transmittance mode imaging in the mid infrared range $\left(4,000-700 \mathrm{~cm}^{-1}\right)$ at $4 \mathrm{~cm}^{-1}$ spectral resolution and $\sim 7 \mu \mathrm{m}$ spatial resolution (Spotlight 400 FTIR Imaging System; PerkinElmer, Waltham, Mass., USA).

FTIRI compositional parameters that are associated with bone quality include: (1) the mineral-to-matrix ratio, which corresponds to the ash weight of bone [Pienkowski et al., 1997]; (2) the carbonate-to-phosphate ratio, which represents the carbonate portion of the mineral content; (3) crystallinity, which correlates to the size and perfection in the $\mathrm{c}$-axis direction as determined by X-ray diffraction [Gadaleta et al., 1996], and (4) the collagen crosslink ratio which is related to the maturity of the collagen [Atti et al., 2002; Paschalis et al., 2001]. Images were collected for 3 randomly selected trabeculae in 3 sections per animal $(n=9)$. ISys software [Malvern Instruments (formerly Spectral Dimensions), Olney, Md., USA) was used to process images as detailed elsewhere [Gourion-Arsiquaud et al., 2008]. Output from the ISys software included color-coded images, the number of pixels in the image, the mean and standard deviation for the parameter imaged, and histograms for each parameter describing its distribution in the trabeculae. A heterogeneity parameter, defined as the full width at half maximum of the Gaussian fit to the distribution histogram was also calculated [Boskey et al., 2009]. Values were averaged for each treatment group and comparisons between groups made using ANOVA, with differences tested by a nested t test.

\section{Results}

The sheep experienced no difficulty with the diet and gained weight during the study year. Several sheep were euthanized due to blockage of the cannula or, in the case of 1 sheep, predation. Thus, at the conclusion of the study there were 4 vehicle-treated MA sheep, 4 RLX-treated MA sheep, and 2 MA-treated ALN sheep.

Relative to normal controls, BMD DXA values decreased at 6 and 12 months in untreated MA ewes (vehicle) and increased relative to the original DXA value in treated MA sheep sacrificed between 10 and 12 months $\left({ }^{*} \mathrm{p}<0.001\right)$, with the smallest increase in RLX-treated sheep [p $<0.05$, ALN vs. RLX at study conclusion (data not shown)]. To enable comparisons, mean FTIRI param-

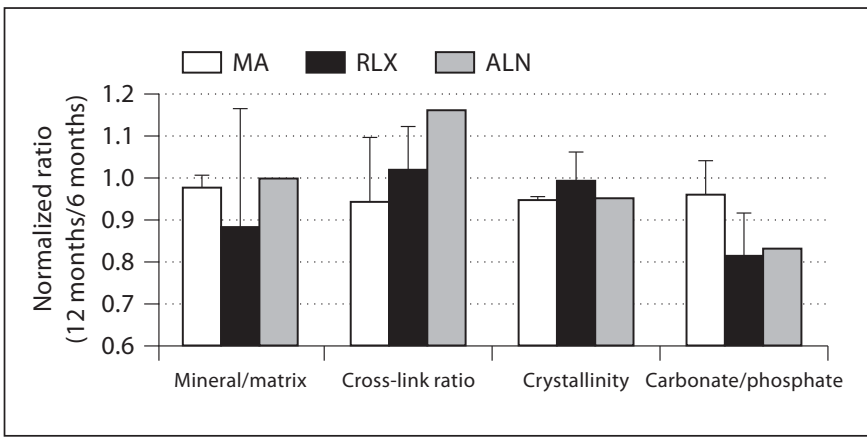

Fig. 1. Mean values of FTIRI-derived parameters for the treatment groups at 12 months relative to non-treated MA sheep at 6 months (MA, vehicle). A value of 1.0 indicates no change from mean of animals in the designated group at 6 months (MA). At 12 months there were 4 sheep in the MA (vehicle) and RLX groups and 2 in the ALN group, preventing statistical comparisons in that group. Parameters shown are the carbonate/phosphate ratio $(\mathrm{cm})$, the mineral/matrix ratio $(\mathrm{mm})$, crystal size and perfection (xy), and collagen maturity. Note that based on the small data set none of the treatments prevented the decrease in carbonate content, but ALN prevented loss of mineral content and changes in crystallinity while leading to an increase in collagen maturity; RLX decreased the mineral/matrix ratio, and normalized crystallinity and collagen maturity. ${ }^{*} \mathrm{p}<0.05$ relative to 6 -month MA.

eters from sheep that survived the study at 12 months were normalized to the mean 6 -month data (fig. 1). The mineral/matrix ratio after 6 months was decreased relative to control sheep, and crystallinity and collagen maturity were increased in untreated MA. Six months of treatment with RLX or ALN tended to decrease the carbonate/phosphate ratio relative to the sheep that received only vehicle (MA) but apparently had no effect on the mineral/matrix ratio. The collagen cross-link ratio tended to exhibit increases for RLX and ALN, with a stronger effect for ALN.

Observation of pixel histograms suggested that heterogeneity was decreased in both treatment groups relative to controls but that vehicle treated sheep also had decreased heterogeneity. The heterogeneity parameter (full width at half maximum) showed nonsignificant distribution changes for ALN and RLX treatment that were not observed in the nontreated vehicle control sheep after 6 months (fig. 2). While no values were significantly different due to the small sample size, ALN treatment seemed to result in broader distributions for the mineral/ matrix ratio and for the carbonate/phosphate ratio relative to the osteopenic MA sheep, while RLX-treated sheep appeared to have narrower distributions. 


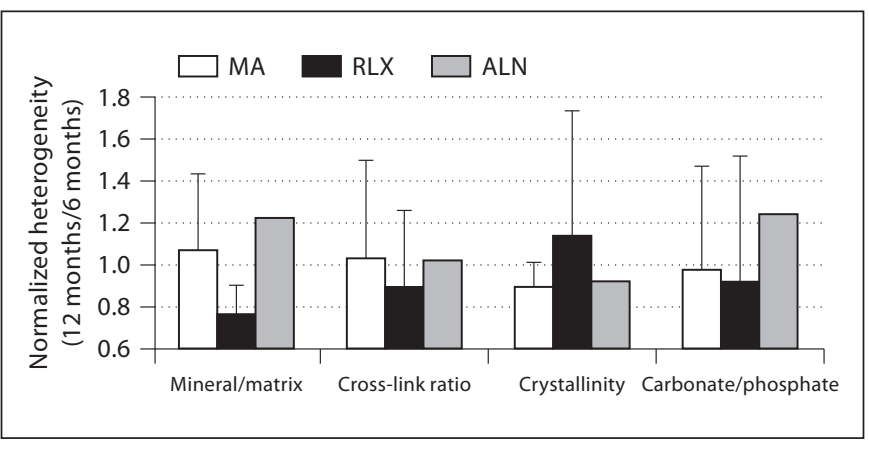

Fig. 2. Heterogeneity of FTIRI-derived parameters for treatment groups at 12 months relative to nontreated controls at 6 months (vehicle). Mean values for treatment groups at 12 months relative to non-treated MA sheep at 6 months (MA, vehicle). A value of 1.0 indicates no change from the mean of animals in designated group at 6 months (MA). At 12 months there were 4 sheep in the MA (vehicle) and RLX groups and 2 in the ALN group. Parameters shown are the carbonate/phosphate ratio $(\mathrm{cm})$, the mineral/ matrix ratio $(\mathrm{mm})$, crystal size and perfection (xy), and collagen maturity. ${ }^{*} \mathrm{p}<0.05$ relative to 6 -month MA.

\section{Discussion}

This preliminary study suggests the MA sheep are a good model for the study of compositional changes during treatment for osteopenia. Despite the small number of animals, compositional parameters changed in manners expected from the treatment modality. The DXA data showed statistically significant mineral loss at 12 months in the sheep given no treatment, but this loss was not detected in sheep given RLX and ALN; this is in agreement with short-term studies in humans [Bauer et al., 2004; Faibish et al., 2006].

Relative to normal control sheep, induction of osteopenia by MA caused a sharpening of the pixel histo- grams for each of the parameters, most likely associated with the loss of newly formed bone and the persistence of older crystals, as is seen in human osteoporosis [Gourion-Arsiquaud et al., 2009]. After 6 months of treatment with ALN, RLX, or vehicle, in the histograms for the treated groups the mineral:matrix peak appeared to get sharper for both groups and was noticeably sharper for the RLX group. This agrees with what has previously been reported for BP-treated osteopenic humans [Boskey et al., 2009] and normal dogs [Gourion-Arsiquaud et al., 2010]. Compared to MA, the peaks in the FTIRI of treated animals were generally broadened. We had anticipated that with new matrix formation, as is seen in the RLX-treated group (reduced mineral/matrix ratio and increased collagen maturity), the distribution would be significantly broadened; however, the small sample size may have prevented such observations. With ALN treatment, where both remodeling and new formation are inhibited, the observed increase in the mineral/matrix ratio, while not significant, was expected. These results suggest that both treatments may restore some bone mass after bone loss and indicate that RLX may provide a unique type of treatment not typically seen with $\mathrm{BP}$ treatment.

\section{Acknowledgments}

This study was supported by NIH Grants AR041325, AR046121, and NSF/CUNY/AGEP-0450830. The authors are grateful to Dr. Simon Turner of Colorado State University for allowing the sheep study to be performed at his facility and for providing the specimens for this study, to the Orthopaedic Residents at the Hospital for Special Surgery who assisted with the sheep dissection, and to the staff of the Analytical Microscopy Core Laboratory who provided the sections used for analysis.

\section{References}

Atti, E., S. Gomez, S.M. Wahl, R. Mendelsohn, E. Paschalis, A.L. Boskey (2002) Effects of transforming growth factor- $\beta$ deficiency on bone development: a Fourier transform-infrared imaging analysis. Bone 31: 675-684.

- Barzel, US (1995) The skeleton as an ion exchange system: implications for the role of acid-base imbalance in the genesis of osteoporosis. J Bone Miner Res 10: 1431-1436.
Bauer, D.C., D.M. Black, P. Garnero, M. Hochberg, S. Ott, J. Orloff, D.E. Thompson, S.K. Ewing, P.D. Delmas, Fracture Intervention Trial Study Group (2004) Change in bone turnover and hip, non-spine, and vertebral fracture in alendronate-treated women: the fracture intervention trial. J Bone Miner Res 19: $1250-1258$.

Bonnelye, E., J.E. Aubin (2005) Estrogen receptor-related receptor alpha: a mediator of estrogen response in bone. J Clin Endocrinol Metab 90: 3115-3121.
Boskey, A.L., R. Coleman (2010) Aging and bone. J Dent Res 89: 1333-1348.

Boskey, A.L., R. Mendelsohn (2005) Infrared spectroscopic characterization of mineralized tissues. Vib Spectrosc 38: 107-114.

Boskey, A.L., L. Spevak, R.S. Weinstein (2009) Spectroscopic markers of bone quality in alendronate-treated postmenopausal women. Osteoporos Int 20: 793-800.

Cummings, S.R., D. Bates, D.M. Black (2002a) Clinical use of bone densitometry: scientific review. JAMA 288: 1889-1897. 
Cummings, S.R., D.B. Karpf, F. Harris, H.K. Genant, K. Ensrud, A.Z. LaCroix, D.M. Black (2002b). Improvement in spine bone density and reduction in risk of vertebral fractures during treatment with antiresorptive drugs. Am J Med 112: 281-289.

Durchschlag, E., E.P. Paschalis, R. Zoehrer, P. Roschger, P. Fratzl, R. Recker, R. Phipps, K. Klaushofer (2006) Bone material properties in trabecular bone from human iliac crest biopsies after 3- and 5-year treatment with risedronate. J Bone Miner Res 21: 1581-1590.

Faibish, D., S.M. Ott, A.L. Boskey (2006). Mineral changes in osteoporosis: a review. Clin Orthop Relat Res 443: 28-38.

Gadaleta, S.J., E.P. Paschalis, F. Betts, R. Mendelsohn, A.L. Boskey (1996). Fourier transform infrared spectroscopy of the solutionmediated conversion of amorphous calcium phosphate to hydroxyapatite: new correlations between X-ray diffraction and infrared data. Calcif Tissue Int 58: 9-16.

Goldstein, S.A. (1987). The mechanical properties of trabecular bone: dependence on anatomic location and function. J Biomech 20: 1055-1061.
Gourion-Arsiquaud, S., M.R. Allen, D.B. Burr, D. Vashishth, S.Y. Tang, A.L. Boskey (2010) Bisphosphonate treatment modifies canine bone mineral and matrix properties and their heterogeneity. Bone 46:666-672.

Gourion-Arsiquaud, S., D. Faibish, E. Myers, L. Spevak, J. Compston, A. Hodsman, E. Shane, R.R. Recker, E.R. Boskey, A.L. Boskey (2009) Use of FTIR spectroscopic imaging to identify parameters associated with fragility fracture. J Bone Miner Res 24: 1565-1571.

Gourion-Arsiquaud, S., P.A. West, A.L. Boskey (2008). Fourier transform-infrared microspectroscopy and microscopic imaging. Methods Mol Biol 455: 293-303.

Harris, S.S., M.J. Wood, B. Dawson-Hughes (1995) Bone mineral density of the total body and forearm in premenopausal black and white women. Bone 16: S311-S315.

MacLeay, J.M., J.D. Olson, R.M. Ennis, C.M. Les, C.A. Toth, D.L. Wheeler, A.S. Turner (2004a) Dietary-induced metabolic acidosis decreases bone mineral density in mature ovariectomized ewes. Calcif Tissue Int 75: 431-437.

Macleay, J.M., J.D. Olson, A.S. Turner (2004b) Effect of dietary-induced metabolic acidosis and ovariectomy on bone mineral density and markers of bone turnover. J Bone Miner Metab 22: 561-568.
Mendelsohn, R., A.L. Boskey, E.P. Paschalis (2005) Infrared microscopy and imaging of hard and soft tissues: applications to bone, skin, and cartilage; in Levin, R.B.I. (ed): Spectrochemical analysis using infrared multichannel detectors. Ames, Blackwell pp 234-260.

Newman, E., A.S. Turner, J.D. Wark (1995). The potential of sheep for the study of osteopenia: current status and comparison with other animal models. Bone 16: 277S-284S

Paschalis, E.P., K. Verdelis, S.B. Doty, A.L. Boskey, R. Mendelsohn, M. Yamauchi (2001) Spectroscopic characterization of collagen cross-links in bone. J Bone Miner Res 16: 1821-1828.

Pienkowski, D., T.M. Doers, M.C. MonierFaugere, Z. Geng, N.P. Camacho, A.L. Boskey (1997) Calcitonin alters bone quality in beagle dogs. J Bone Miner Res 12: 1936-1943.

Turner, A.S. (2002) The sheep as a model for osteoporosis in humans. Vet J 163: 232-239.

Turner, R.T., A. Maran, S. Lotinun, T. Hefferan, G.L. Evans, M. Zhang, J.D. Sibonga (2001) Animal models for osteoporosis. Rev Endocr Metab Disord 2: 117-127.

Wiederkehr, M., R. Krapf (2001) Metabolic and endocrine effects of metabolic acidosis in humans. Swiss Med Wkly 131: 127-132. 